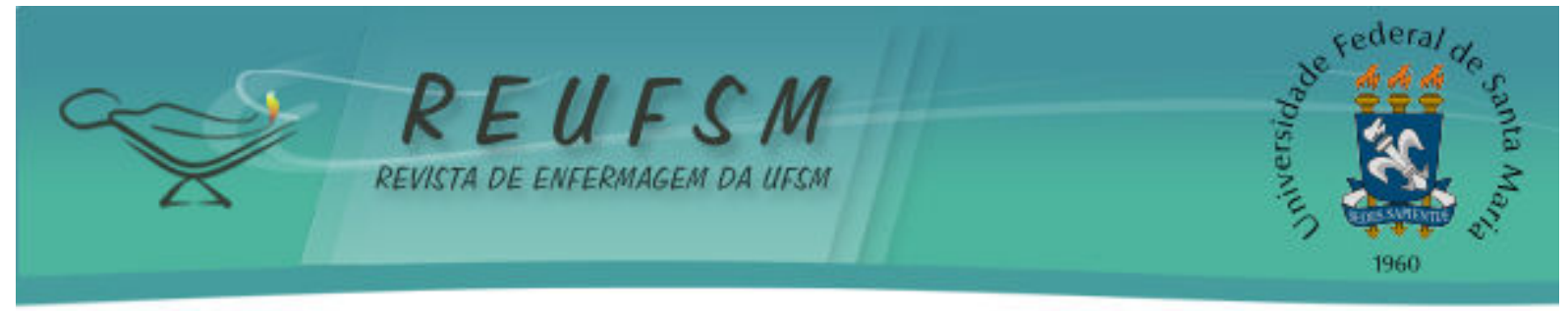

ARTIGO ORIGINAL

\title{
ENFERMAGEM E CULTURA: CARACTERÍSTICAS DAS TESES E DISSERTAÇÕES PRODUZIDAS NA PÓS-GRADUAÇÃO DA ENFERMAGEM BRASILEIRA
}

\section{NURSING AND CULTURE: CHARACTERISTICS OF THESES AND DISSERTATION PRODUCED IN BRAZILIAN NURSING POSTGRADUATE PROGRAMS}

\section{ENFERMERÍA Y CULTURA: CARACTERÍSTICAS DE LAS TESIS Y DISERTACIONES PRODUCIDAS EN EL POSTGRADO DE LA ENFERMERÍA BRASILEÑA}

\author{
Fabiano Oliveira Antonini ${ }^{1}$ \\ Astrid Eggert Boehs ${ }^{2}$ \\ Maria Helena Lenardt ${ }^{3}$ \\ Maria de Lourdes Denardin Budó ${ }^{4}$ \\ Marisa Monticelli ${ }^{5}$
}

Doi: $10.5902 / 217976929724$

RESUMO: Objetivo: identificar as características das teses e dissertações relacionadas à enfermagem e cultura, alicerçadas na antropologia. Método: pesquisa bibliográfica do período de 1987 a 2011. As informações foram organizadas em um instrumento com categorias prédefinidas. A análise foi descritiva e interpretada à luz de estudos que englobam os temas enfermagem e cultura. Resultados: é possível identificar uma curva ascendente na produção, mantendo-se crescente até os dias atuais. O tema predominante foi Saúde da Mulher, o hospital é o local de estudo mais citado. O referencial mais utilizado foi a Teoria de Leininger. As pesquisas apresentam consonância com os temas propostos pelos órgãos governamentais e também organizativos da enfermagem brasileira. Ainda faltam pesquisas relacionadas com os povos indígenas, população negra e portadores de necessidades especiais. Conclusão: a enfermagem brasileira tem amplas possibilidades para avançar, tornando mais visível esta área.

Descritores: Enfermagem; Cultura; Pesquisa em enfermagem.

ABSTRACT: Aim: to identify characteristics of theses and dissertations related to nursing and culture, grounded in anthropology. Method: bibliographic research, of the period 1987-2011. The information was organized into an instrument with predefined categories. The analysis was descriptive and interpreted in the light of studies that encompass the themes nursing and culture. Results: it is possible to identify an upward curve in production, keeping growing until the present day. The predominant theme was Women's Health, the hospital being the most cited study site. The benchmark used was the Theory Leininger. The present research consistent with the themes proposed by the government and also organizational Brazilian nursing, there are still research related to indigenous peoples, black population and people with special needs. Conclusion: Brazilian nursing has ample opportunities to advance, making this area more visible.

Descriptors: Nursing; Culture; Nursing research.

RESUMEN: Objetivo: identificar las características de las tesis y disertaciones, relacionadas a la

\footnotetext{
${ }^{1}$ Enfermeiro do Trabalho. Mestre em Enfermagem. Instituto Federal de Educação Ciência e Tecnologia de Santa Catarina. Florianópolis, Santa Catarina, Brasil. fabiano.antonini@gmail.com

${ }^{2}$ Enfermeira. Doutora em Enfermagem. Universidade Federal de Santa Catarina. Florianópolis. Santa Catarina. Brasil. astridboehs@hotmail.com

${ }^{3}$ Enfermeira. Doutora em Enfermagem. Universidade Federal do Paraná. Curitiba. Paraná. Brasil. curitiba.helena@gmail.com

${ }^{4}$ Enfermeira. Doutora em Enfermagem. Universidade Federal de Santa Maria. Santa Maria. Rio Grande do Sul, Brasil. lourdesdenardin@gmail.com

${ }^{5}$ Enfermeira. Doutora em Enfermagem. Universidade Federal de Santa Catarina. Florianópolis. Santa Catarina. Brasil. marisa@nfr.ufsc.br
} 


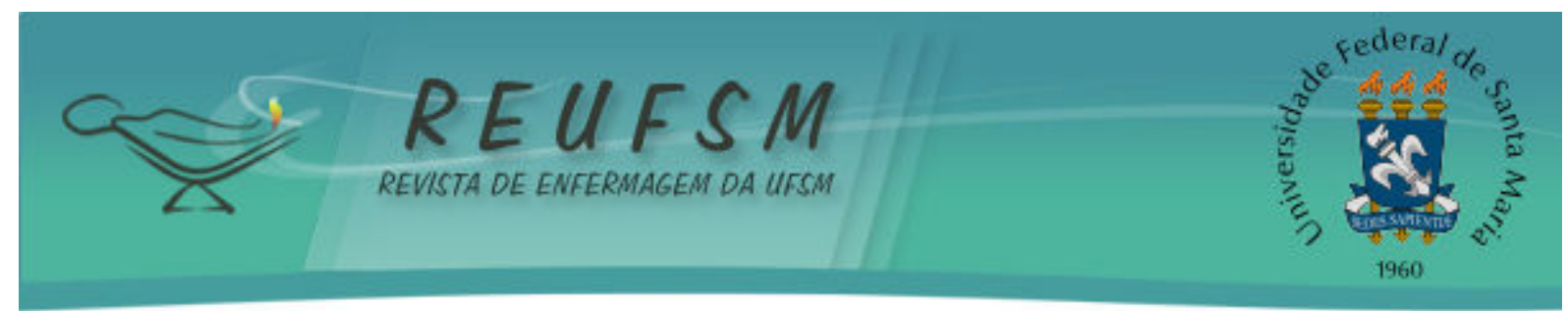

enfermería y la cultura, basada en la antropología.Método: investigación bibliográfica, del período 1987-2011. Las informaciones fueron organizadas en un instrumento con categorías predefinidas. El análisis fue descriptivo e interpretado a la luz de los estudios que engloban los temas de enfermería y la cultura. Resultados: es posible identificar una curva ascendente en la producción, manteniéndose crecente hasta los días actuales. El tema predominante fue la Salud de la Mujer, el hospital es el lugar de estudio más citado. La referencia más utilizada fue la Teoría de Leininger. Las investigaciones presentan consonancia con los temas propuestos por el gobierno y también organizativos de la enfermería brasileña. Aún no hay investigaciones relacionadas con los pueblos indígenas, población negro y los que tienen necesidades especiales. Conclusión: la enfermería brasileña tiene amplias oportunidades para avanzar, haciéndose más visible esta área.

Descriptores: Enfermería; Cultura; Investigación en enfermería

\section{INTRODUÇÃO}

Desde a implantação do Sistema Único de Saúde (SUS) esforços vêm sendo desenvolvidos para sua efetiva consolidação. Foram criadas estratégias no sentido de integrar os diferentes níveis de atenção e, sobretudo, valorizar a assistência primária de saúde com a Estratégia de Saúde da Família. Dentro desta estratégia, a equipe de saúde trabalha num território próximo do local onde as pessoas vivem. A enfermagem integra essa equipe, na qual o enfermeiro exerce, com frequência, a liderança sobre os agentes comunitários de saúde, que são aqueles atores diretamente vinculados à diversidade da população.

Assim, é necessário que os profissionais de enfermagem se preocupem em prestar cuidado não apenas voltado para a manifestação biológica das doenças. 0 cuidado deve estar associado com as condições econômicas, sociais, culturais e do ambiente onde os indivíduos e suas coletividades estão inseridos, pois os profissionais de saúde, principalmente os enfermeiros, se defrontam de forma muito estreita com as características da população brasileira. População que é miscigenada, composta por pessoas de diferentes classes sociais, religiões, origens étnicas, de gênero, e apresentando uma diversidade cultural que permeia as relações de saúde e doença, incluindo a adesão a tratamentos e cuidados.

A realidade da enfermagem brasileira é campo para implementação de cuidados tendo em vista a diversidade regional de contextos sociais e culturais que suscita distintas necessidades, significados e expectativas de cuidado. No entanto, na prestação do cuidado essas diferenças, frequentemente, são pouco consideradas. 0 sistema de atenção à saúde não está desacoplado de outros aspectos gerais da cultura, uma vez que a mesma envolve elementos sobre os quais os atores sociais constroem significados para as ações e interações sociais concretas e temporais, sustentando as formas sociais vigentes, as instituições e seus modelos operativos. ${ }^{1}$

Cabe ainda considerar que a organização da assistência nos níveis de atenção à saúde deve fazer a interlocução com temáticas antropológicas e sociais, entendendo-se os sentidos atribuídos pelos sujeitos aos eventos de saúde e doença, políticas públicas e os serviços de saúde. ${ }^{2}$ Dessa forma, considerar a importância da cultura na atuação de enfermagem e de outros profissionais de saúde exige o desenvolvimento de pesquisas, no meio acadêmico, com o objetivo de construir fundamentos para a prática assistencial. Esse desafio para a enfermagem brasileira depende muito dos conhecimentos produzidos pelos Programas de Pós-Graduação em Enfermagem, que têm crescido rapidamente no Brasil, e têm contribuído para a formação dos profissionais que irão atuar no SUS.

No que se refere especificamente aos estudos de enfermagem relacionados com o tema cultura, cabe ressaltar que, em 1985, Madeleine Leininger apresentou, pela primeira 


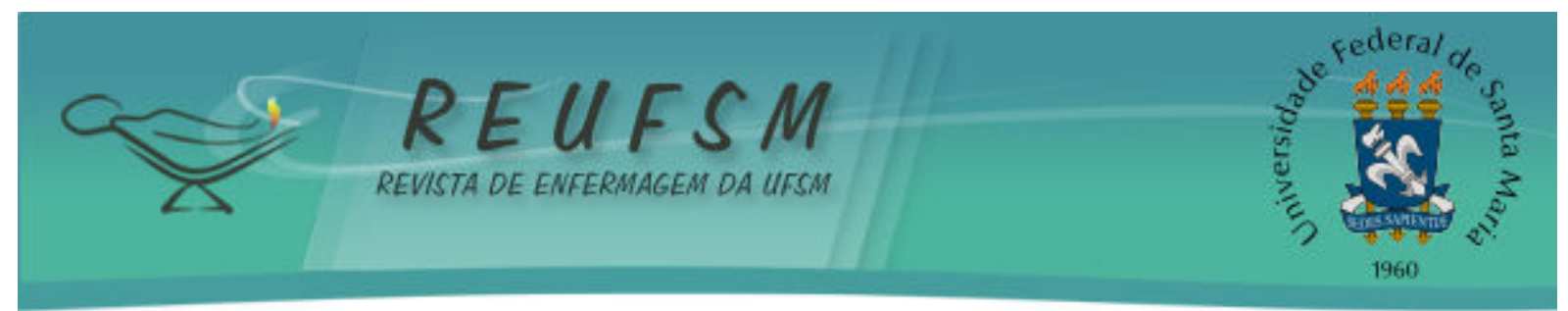

vez no Brasil, a Teoria da Diversidade e Universalidade do Cuidado Cultural (TUDCC). Na época, a difusão de outros autores da antropologia da saúde, no âmbito da pós-graduação, como o americano Arthur Kleinmann, que defende a ideia dos sistemas de cuidado como sendo sistemas culturais, motivaram ainda mais a busca por esse conhecimento. ${ }^{3}$ Alguns estudos foram realizados para analisar as pesquisas relacionadas a este tema, porém, foram delimitados somente para os que utilizaram a TUDCC. ${ }^{4-8}$

A pergunta que norteou a pesquisa foi: o que a enfermagem pesquisou sobre 0 tema enfermagem e cultura com base na antropologia, tendo por objetivo identificar as características das teses e dissertações relacionadas à enfermagem e cultura, alicerçadas na antropologia.

\section{MÉTODO}

Realizou-se pesquisa bibliográfica, cujas fontes foram resumos de dissertações de mestrado e teses de doutorado dos Programas de Pós-Graduação de Enfermagem brasileiros, abrangendo o período de 1987-2011. Esse recorte temporal se justifica por ser o período pós-presença da pesquisadora e teórica Madeleine Leininger no Brasil, incentivando as enfermeiras brasileiras a investigarem sobre o tema cuidado e cultura, bem como pelo fato de o Banco de Teses da Coordenação de Aperfeiçoamento de Pessoal de Nível Superior (CAPES) disponibilizar os resumos de teses e dissertações defendidas a partir de 1987.

Para a coleta de dados foram utilizados os critérios de inclusão: dissertações e teses de enfermagem que contemplavam a definição de cultura, fundamentada em conceitos da antropologia, e que foram desenvolvidas nos programas de pós-graduação de enfermagem brasileiros.

Os dados foram acessados através do link da CAPES (http://capesdw.capes.gov.br/capesdw/), que fornece acesso a uma ferramenta de consulta, por meio das palavras-chave "enfermagem" e "cultura", de 01/09/2011 a 30/04/2012. Inicialmente foram encontradas 474 dissertações e teses. Porém, após leitura e análise das informações de identificação e resumo, foram descartadas 350 , por não se enquadrarem nos critérios sendo selecionados 124 para o estudo.

Após essa definição, foi desenvolvido um instrumento de coleta e organização dos dados, com os seguintes itens: autor, título, instituição de ensino, estado de origem, tese ou dissertação, orientador, grupo de pesquisa, ano de defesa, objetivos, local de estudo, população, metodologia e referencial teórico.

Todos os dados foram registrados em planilhas próprias no Programa Excel, com o auxílio de filtros, que estão disponíveis neste mesmo programa com a finalidade de realizar a análise descritiva dos dados, em relação às características da produção das teses e dissertações, no que diz respeito à frequência e porcentagem. Tais dados foram discutidos com estudos publicados, relacionados à produção de conhecimento, envolvendo enfermagem e cultura.

Este estudo foi submetido ao Comitê de Ética em Pesquisa com Seres Humanos da Universidade Federal de Santa Catarina (UFSC), com parecer aprovado sob número 2.085, em 03 de outubro de 2011.

\section{RESULTADOS E DISCUSSÃO}

As dissertações de mestrado e teses de doutorado foram desenvolvidas em 11 dos 26 Estados e Distrito Federal, distribuídos nas Regiões Nordeste, Centro-oeste, Sudeste e Sul, sendo que a Região Norte não apresentou estudos. 


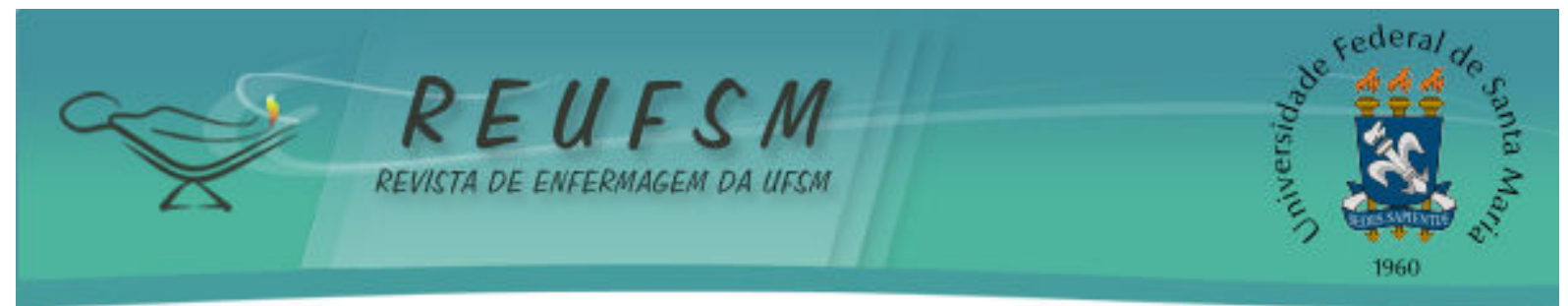

A figura 1 mostra a desigualdade na distribuição da produção de conhecimento nessa área no Brasil, com a produção centrada nas Regiões Sudeste e Sul. Alguns estados de fronteiras e a região Norte do Brasil, nos quais há grande diversidade cultural, mas que ainda estão iniciando Programas de sua Pós-graduação stricto sensu, não possuem produção de teses e dissertações sobre esse tema.

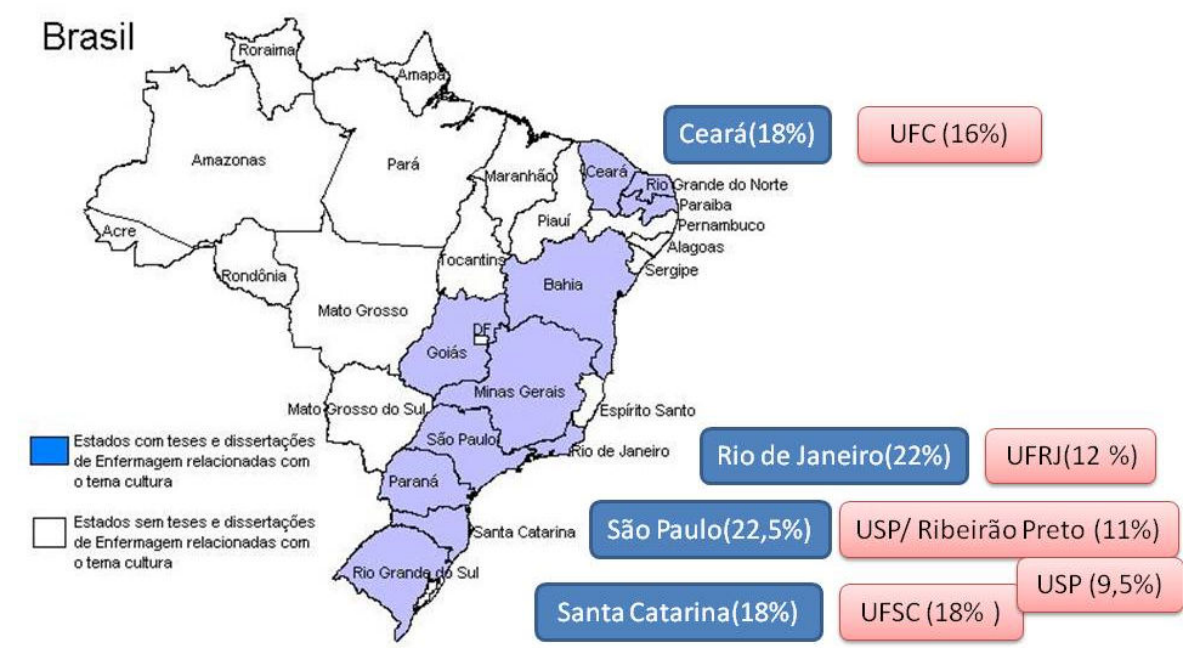

Figura 1 - Distribuição de teses e dissertações de enfermagem relacionadas ao tema cultura nos Estados Brasileiros, 1987-2011.

Fonte: produção dos autores.

Ainda, concernente aos estados de origem, na figura 1 observa-se os que se destacaram na produção de conhecimento nessa área: São Paulo com 28 (22,5\%), Rio de Janeiro 27 (22\%), Santa Catarina, com 22 (18\%) e Ceará, com 22 (18\%).

Quanto às instituições, em que foram desenvolvidas as dissertações e teses relacionando enfermagem e cultura, destacaram-se a Universidade Federal de Santa Catarina com 18\% dos resumos, Universidade Federal do Ceará (16\%), Universidade Federal do Rio de Janeiro (12\%), Universidade de São Paulo/Ribeirão Preto (11\%), e a Universidade de São Paulo (9,5\%). Em menores percentuais foram identificadas dissertações e teses desenvolvidas pelos Programas de Pós-Graduação das universidades públicas, federais ou estaduais, nos estados do Rio Grande do Sul, Paraná, Minas Gerais, Goiás, Bahia, Paraíba e Rio Grande do Norte.

Nos demais Estados, onde não foram identificadas dissertações nem teses nessa área, pode-se inferir que a causa seja pela ausência de pesquisadores que estudem essa temática, ou porque os programas não possuem esta linha de pesquisa. Tais resultados estão em consonância com uma pesquisa acerca de teorias de enfermagem, a qual apontou que a maior produção se originava no Sul, Sudeste e Nordeste do país. ${ }^{9}$

A seguir, na figura 2, apresenta-se o ano da publicação das dissertações e teses. 

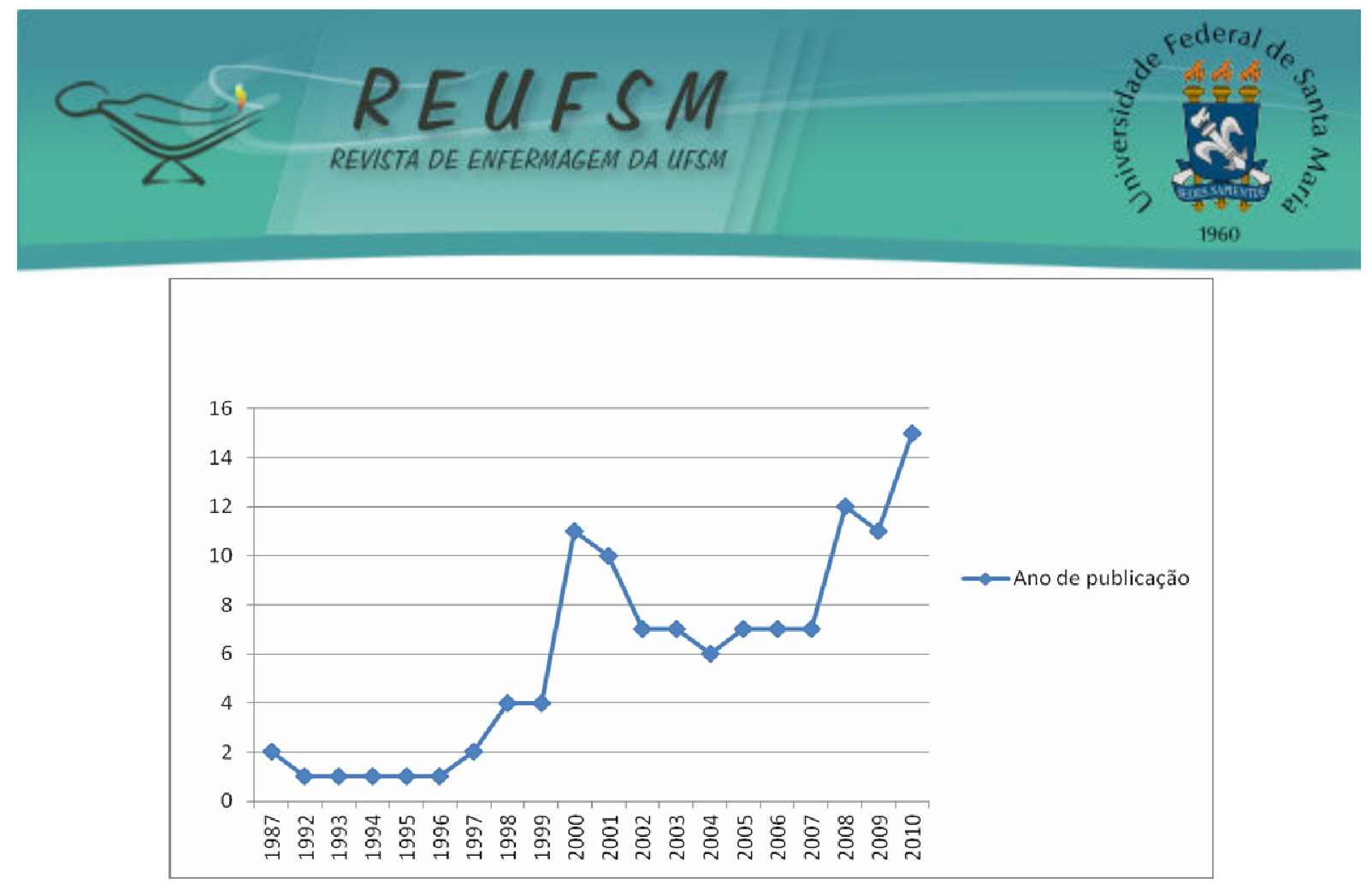

Figura 2 - Ano de publicação das dissertações e teses de enfermagem relacionadas ao tema cultura, 1987-2011.

Referente ao ano de publicação foi possível identificar uma curva ascendente das dissertações e teses da pós-graduação em enfermagem. Apresenta um pico maior no ano de 2000, houve queda entre 2001 a 2008 e depois cresceu novamente a partir de 2009 e mantendo-se até os dias atuais (Figura 2). Esse crescimento das publicações está relacionado com o incremento da oferta de cursos de Pós-graduação em Enfermagem. Sabe-se que, com a política de expansão do Sistema Nacional de Pós-Graduação, na década de 1990, evoluiu significativamente o número de formados nas diferentes áreas de conhecimento, em especial, na enfermagem. ${ }^{10}$ Quanto ao crescimento dos cursos de doutorado abrangendo todas as áreas de conhecimento, no período entre 1996 e 2004, a área de Ciências da Saúde foi a que mais cresceu. Só no ano de 2005 houve um crescimento de $31,25 \%$ em relação à avaliação trienal $1998-2000 .^{10}$

0 percentual de dissertações foi significativamente maior (72\%), quando comparado às teses (28\%). Pode-se afirmar que a relação entre dissertações e teses é diretamente proporcional ao número de cursos de mestrado e doutorado em enfermagem ofertados no Brasil ${ }^{11}$, com predominância de cursos de mestrado, acadêmicos ou profissionais (56 cursos), sobre os de doutorado (27 cursos).

No que diz respeito aos orientadores das teses e dissertações, apenas dois grupos tinham associação direta com a temática enfermagem e cultura: Grupo de pesquisa Sociedade, Cultura e o Processo Saúde-Doença da Universidade de São Paulo (USP), e Cultura e Humanização do Cuidado da Universidade de Fortaleza (UNIFOR). Os demais orientadores, pertencem a grupos de pesquisa de temas variados e não diretamente relacionados ao tema cultura. Infere-se que tal situação contribui para que o tema cultura, na enfermagem, apresente um importante déficit de visibilidade, apesar da sua extensa produção desde a década de 1980 .

Os locais de desenvolvimento das dissertações e teses foram: hospital (23\%), domicílio (13\%), unidades básicas de saúde (18\%), maternidade $(11 \%)$, grupo/casa de apoio $(9 \%)$, comunidade $(6 \%)$, escola e creches $(5 \%)$, ambulatório (4\%) e penitenciária (1\%). Não foram identificados os locais de estudo em $24(17 \%)$ dos resumos analisados. 0 que chama atenção é que em estudos relacionados com a cultura, a descrição do local de realização deveria constar nos resumos. Na perspectiva da cultura do ponto de vista antropológico, deve-se considerar determinado grupo de pessoas ou comunidade, para estudar valores aprendidos, 


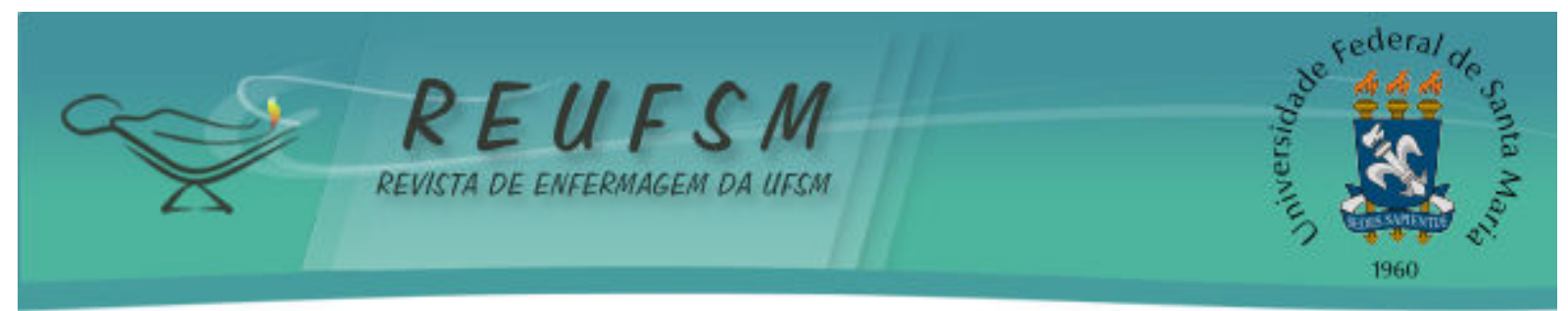

compartilhados e transmitidos; crenças, normas e estilos de vida que orientam o pensamento; decisões e ações de forma padronizada no grupo em que as pessoas estão inseridas. ${ }^{12}$

Dentre os temas abordados nas dissertações e teses salienta-se que $19,5 \%$ pesquisaram questões relacionados à gestação e puerpério, 19\% investigaram itinerários terapêuticos e 10,5\% abordaram práticas e cuidados que, juntos, somam $49 \%$ dos temas abordados.

A saúde da mulher foi a temática mais investigada; predominância essa que pode estar relacionada ao fato de as primeiras linhas de pesquisa adotadas nos Programas de PósGraduação, criados nos anos de 1970, referirem-se às áreas de concentração na saúde da mulher. ${ }^{10}$ Identificou-se uma preocupação, ainda incipiente, na realização de estudos relacionando a cultura com a saúde dos homens e idosos, sendo áreas com atenção mais atual.

A Agenda Nacional de Prioridades de Pesquisa em Saúde do Ministério da Saúde ${ }^{13}$ tem como pressuposto apresentar as necessidades nacionais e regionais de pesquisa em saúde. Das 24 necessidades de pesquisa propostas nessa agenda, 11 foram identificadas nos resumos: saúde mental, violência, acidentes e trauma, saúde do idoso, saúde da criança e do adolescente, saúde da mulher, alimentação e nutrição, promoção da saúde, doenças não transmissíveis, doenças transmissíveis e pesquisa clínica. Cabe ressaltar a existência de uma lacuna na produção de conhecimento relacionando enfermagem e cultura em temas propostos nessa agenda, como: saúde dos povos indígenas, saúde da população negra e saúde dos portadores de necessidades especiais. ${ }^{13}$

Também considerando a Política para a Enfermagem Brasileira em Ciência, Tecnologia e Inovação ${ }^{14}$ constata-se que os temas das teses e dissertações estão ligados a linhas temáticas desse documento. Principalmente no que se refere às práticas avançadas de cuidado de enfermagem direcionadas aos quatro grupos humanos: criança, adolescente, adulto (homem e mulher) e idoso; e gestão e organização do sistema de produção do cuidado de enfermagem.

Os tipos de estudo mencionados nas teses e dissertações foram: pesquisa qualitativa (37\%) (sem especificar o método), etnográfico (33\%), exploratório-descritivo $(10 \%)$, estudo de caso $(9 \%)$, pesquisa convergente-assistencial $(4 \%)$ e história de vida $(3 \%)$. Em sete resumos (4\%), não foi mencionado o tipo de estudo. Isto denota que há necessidade de aumentar o rigor na elaboração e na divulgação dos resultados das pesquisas qualitativas uma vez que este tipo de pesquisa busca sua afirmação no mundo científico. Segue na figura 3 o referencial teórico das dissertações e teses.

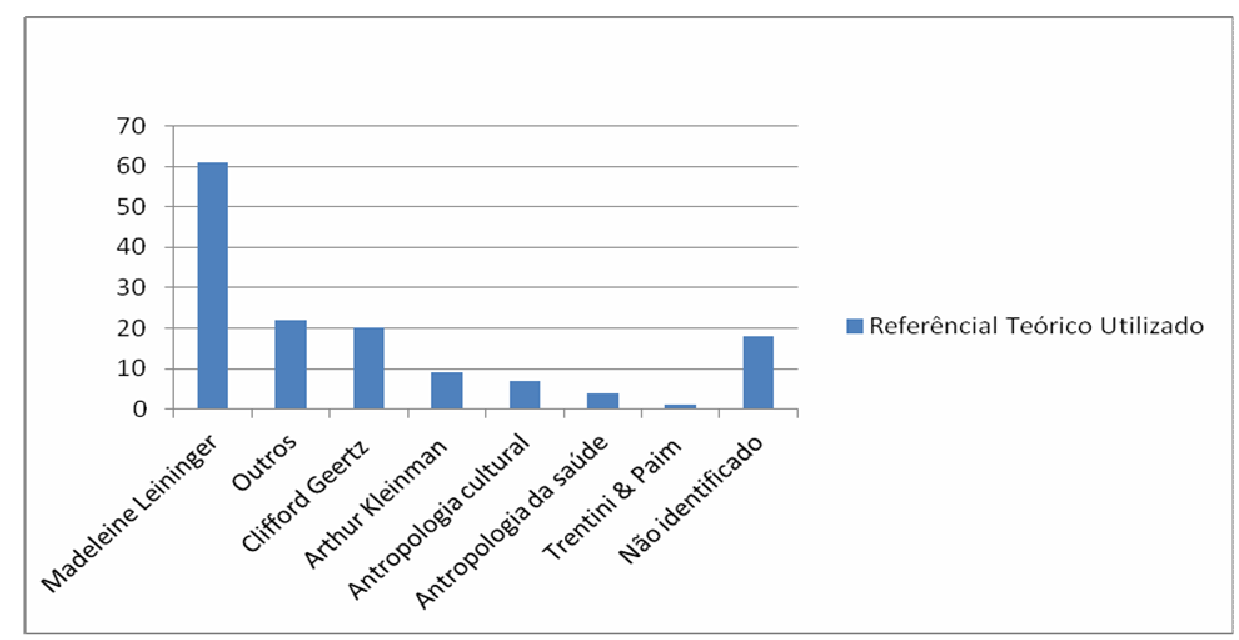

Figura 3 - Referencial teórico empregado nas dissertações e teses de enfermagem relacionadas ao tema cultura, 1987-2011. 


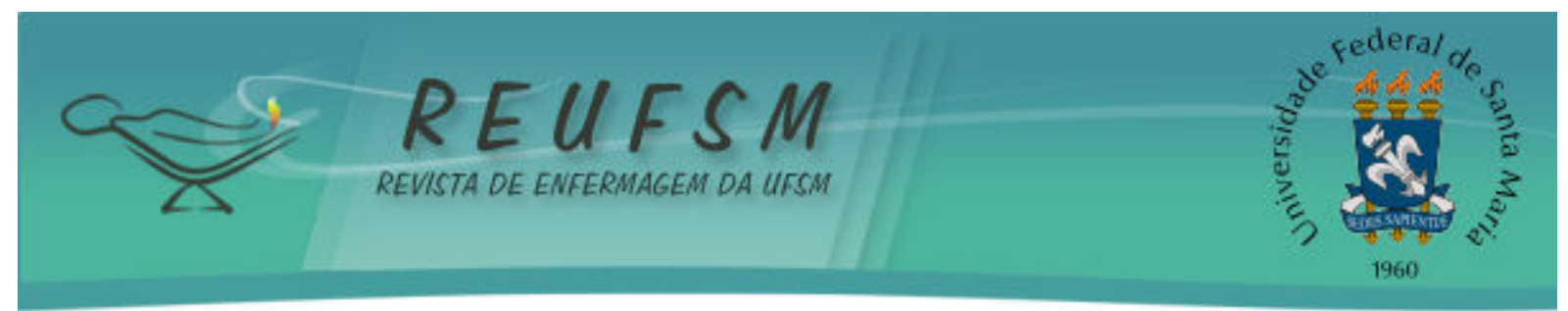

O referencial teórico mais utilizado foi a TUDCC, (43\%), indicando a grande difusão das ideias desta autora na enfermagem brasileira. Outros autores também foram utilizados, como: Clifford Geertz (14\%) e Arthur Kleinman (6\%). Em alguns resumos foi referida a utilização de conceitos da antropologia cultural (6\%) e antropologia da saúde (6\%), sem explicitar os autores. Com número muito menos expressivo foram utilizados os autores Arthur Spradley, Roland Robertson, Mike Featherstone, Hans Georg Gadamer e Michel Maffesoli, que foram agrupados em "outros" e que, juntos, representam 15\% dos suportes teóricos (Figura 3).

Considerando a importância da cultura na atuação da enfermagem, verifica-se que os pesquisadores buscam referenciais para desenvolver suas pesquisas, por meio de pressupostos, conceitos e/ou teorias alicerçadas na antropologia.

Em uma revisão de literatura ${ }^{9}$ sobre teorias de enfermagem publicadas em periódicos da área, entre 1998 e 2007, a TUDCC foi a segunda teoria mais utilizada. Os autores enfatizam que esta teoria desponta como importante referencial, não apenas por permitir a compreensão de que os fenômenos de saúde e doença permeiam os aspectos culturais, mas porque apresenta uma proposta metodológica própria, o que favorece o desenvolvimento de pesquisas.

Estudo ${ }^{5}$ cujo objetivo foi identificar o perfil das dissertações de mestrado em enfermagem desenvolvidas pela UFSC, e que utilizaram a TUDCC em projetos assistenciais, apresentou semelhanças com o presente estudo referente ao ano de defesa, à temática e aos locais de estudo. Ainda outra pesquisa que apresenta o cenário do desenvolvimento de dissertações de mestrado e doutorado no período 1993 a 2004, tendo como fundamentação a TUDCC no Brasil, aponta que a maior densidade dessa produção está nos Programas de Pós-graduação da Universidade Federal de Santa Catarina ${ }^{15}$, também identificado no presente estudo.

0 conjunto destes dados revelam o que também é apontado na literatura ${ }^{16}$ que a enfermagem pode tornar-se consciente da sua cultura profissional e exercer cada vez mais um papel de mediador entre a cultura do sistema profissional, o popular e familiar.

\section{CONSIDERAÇÕES FINAIS}

O estudo caracteriza a produção de conhecimento com uma curva ascendente até o ano 2000, com queda entre 2001 a 2008 e voltando a ascender a partir de 2009, mostrando que há um crescente interesse, por parte de enfermeiros em formação de pósgraduação, com os estudos de Enfermagem relacionados ao tema cultura.

Há desigualdade na distribuição da produção de conhecimento nessa área no Brasil: alguns estados como os da região norte, cuja população apresenta grande diversidade cultural, não possuem estudos considerando os aspectos culturais relacionados à saúde da população.

As dissertações e teses dos Programas de Pós-Graduação em Enfermagem do Brasil, relacionadas à enfermagem e cultura, apresentam consonância com os temas e linhas de pesquisa propostos pela Agenda Nacional de Prioridades de Pesquisa em Saúde do Ministério da Saúde, assim como pela agenda dos órgãos próprios da enfermagem. No entanto ainda há necessidade de ampliar as pesquisas para temas pouco explorados.

Tal constatação faz recomendar que os pesquisadores dos Programas de Pósgraduação em Enfermagem realizem pesquisas relacionado com os temas da saúde dos povos indígenas, saúde da população negra entre outros grupos. Em todos estes temas a abordagem pode estar focada tanto nos usuários como nos profissionais.

Finalmente considera-se que há necessidade de criar grupos de pesquisa específicos sobre cultura e enfermagem tornando esta área mais visível e com condições de dar destaque para este tema. 


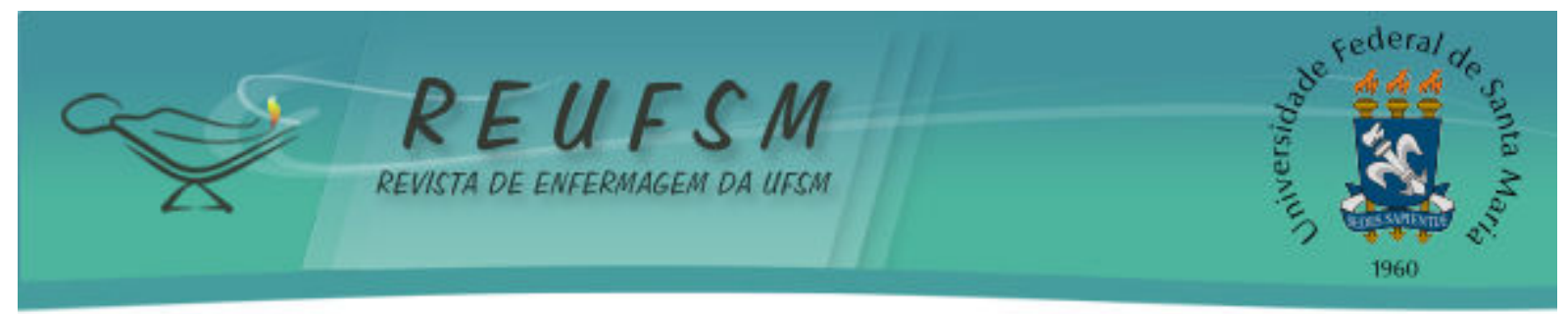

\section{REFERÊNCIAS}

1. Langdon EJ, Wiik FB. Antropologia, saúde e doença: uma introdução ao conceito de cultura aplicado às ciências da saúde. Rev Latinoam Enferm. 2010; 18(3):173-81.

2. Garnelo L, Langdon EJ. A antropologia e a reformulação das práticas sanitárias na atenção básica à saúde. In: Minayo MCS, Coimbra JRC, organizadores. Críticas e atuantes: ciências sociais e humanas em saúde na América Latina. Rio de Janeiro: Editora Fiocruz; 2005. p.143-56.

3. Kleinman A. Patients and healers in the context of culture. Berkeley: University of California Press; 1980.

4. Moura MAV, Chamilco RASI, Silva LR. A teoria transcultural e sua aplicação em algumas pesquisas de enfermagem: uma reflexão. Esc Anna Nery Rev Enferm. 2005; 9(3):434-40.

5. Monticelli M, Boehs AE, Guesser JC, Gehrmann T, Paiva, K. Perfil de dissertações que utilizam a teoria de Leininger vinculadas a um programa de mestrado em enfermagem do sul do país. Ciênc Cuid Saúde. 2008; 4(7):447-53.

6. Monticelli M, Boehs AE, Guesser JC, Gehrmann T, Martins M, Manfrini GC. Aplicações da teoria transcultural na prática da enfermagem a partir de dissertações de mestrado. Texto \& Contexto Enferm. 2010; 19(2):220-8.

7. Boehs AE, Monticelli M, Fernandes GCM, Feyer ISS, Rumor PCF. Conceitos da teoria do cuidado cultural em dissertações de mestrado. Rev RENE. 2010; 11(4):182-9.

8. Seima MD, Michel T, Méier MJ, Wall ML, Lenardt MH. A produção científica da enfermagem e a utilização da teoria de Madeleine Leininger: revisão integrativa 1985 - 2011. Esc Anna Nery Rev Enferm. 2011; 15(4):851-7.

9. Schaurich D, Crossetti MGO. Produção do conhecimento sobre teorias de enfermagem: análise de periódicos da área, 1998-2007. Esc Anna Nery Rev Enferm. 2010; 14(1):182-8.

10. Rodrigues RAP, Erdmann AL, Silva IA, Fernandes JD, AraújoTL, Vianna LAC, et al. Educação do doutorado em enfermagem no Brasil. Rev Latinoam Enferm. 2008; 16(4):665-71.

11. Coordenação de Aperfeiçoamento de Pessoal de Nível Superior. Cursos recomendados e reconhecidos. Brasília; 2012. [acesso em 2012 out. 20]. Disponível em: <http://www.capes.gov.br/avaliacao/cursos-recomendados-e-reconhecidos>.

12. Leininger M, McFarland MR. Culture care diversity and universality: a worldwide nursing theory. New York: McGraw-Hill; 2006.

13. Ministério da Saúde (BR). Agenda Nacional de Prioridades de Pesquisa em Saúde [internet] 2008 [acesso em 2012 jun 11]. Disponível em: <http://bvsmv.saude.gov.br/bvs/publicacoes/agenda_portugues_montado.pdf>.

14. Associação Brasileira de Enfermagem. Política para a enfermagem brasileira em ciência, tecnologia e inovação [online]. Fortaleza: ABEN, 2009 Dez 10 [acesso em 2012 Ago. 14]. Disponível em: http://www.abennacional.org.br/index.php?path=227.

15. Oriá MO, Ximenes LB, Alves MDS. Utilização da teoria do cuidado cultural na pós-graduação em enfermagem: a realidade brasileira. Rev enferm UERJ. 2006; 14(2):245-52.

16. Bittencourt FS, Pereira PL, Gonzaga SM, Boehs AE, Heidemann ISB, Báfica ACMF. Agentes comunitários de saúde: atribuições na saúde da criança. Rev enferm UFSM. [internet] 2011[acesso 2013 Set 3];1(3):318-25. Disponível em: http://cascavel.ufsm.br/revistas/ojs.2.2/index. php/reufsm/issue/view/196. 


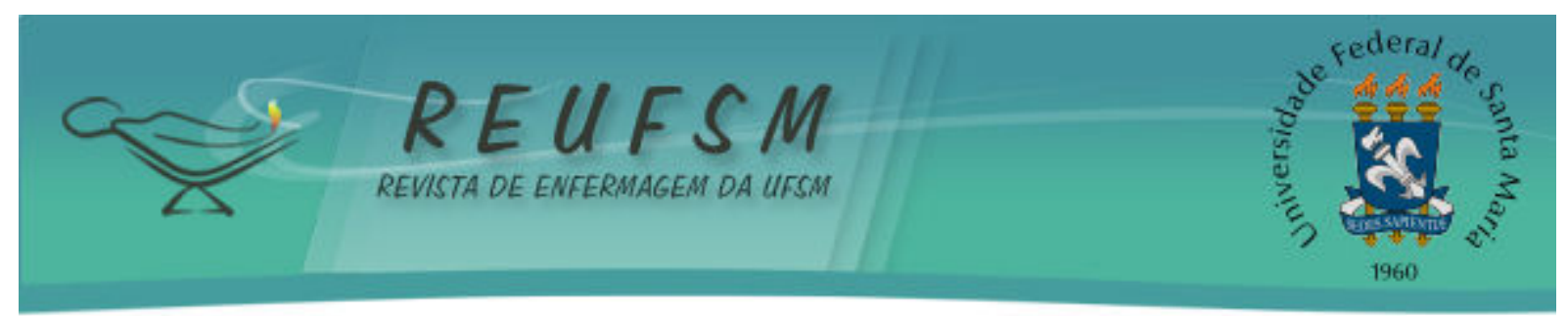

Data de recebimento: 08/07/2013

Data de aceite: 10/02/2014

Contato com autor responsável: Fabiano Oliveira Antonini. Rua. 14 de Julho, 150 - Bairro Coqueiros - CEP 88075-010 - Florianópolis - SC - Brasil. Fone: (48) 38779017 / 38779018. E-mail: fabiano.antonini@gmail.com 